\title{
Efeitos da luz e da temperatura na germinação de sementes de Syngonanthus elegantulus Ruhland, S. elegans (Bong.) Ruhland e S. venustus Silveira (Eriocaulaceae) ${ }^{1}$
}

\author{
Patrícia Gonçalves Oliveira² e Queila Souza Garcia ${ }^{2,3}$
}

Recebido em 14/10/2004. Aceito em 18/02/2005

\begin{abstract}
RESUMO - (Efeitos da luz e da temperatura na germinação de sementes de Syngonanthus elegantulus Ruhland, S. elegans (Bong.) Ruhland e $S$. venustus Silveira (Eriocaulaceae)). O objetivo deste estudo foi avaliar os efeitos da luz e da temperatura na germinação de sementes de Syngonanthus elegantulus Ruhland, S. elegans (Bong) Ruhland e S. venustus Silveira. Os experimentos foram realizados em câmaras de germinação sob luz e escuro contínuos nas temperaturas constantes de $15,20,25,30,35$ e $40{ }^{\circ} \mathrm{C}$ e alternadas de $25-15$, 30-15, 35-15 e $35-25^{\circ} \mathrm{C}$. A germinabilidade foi baixa (36,5\%), sob luz, em S. elegantulus na faixa de 15 a $30^{\circ} \mathrm{C}$ e elevada em S. elegans $(75 \%)$ e $S$. venustus $(67,5 \%)$, na faixa de 20 a $30^{\circ} \mathrm{C}$. A temperatura de $40{ }^{\circ} \mathrm{C}$ inibiu a germinação de todas as espécies. No escuro, não ocorreu germinação de sementes de $S$. elegantulus, enquanto $S$. elegans apresentou valores de percentagem de germinação muito baixos $(11,5 \%)$, a 25 e $30{ }^{\circ} \mathrm{C}$ e S. venustus $(21 \%)$, na faixa de 20 a $35^{\circ} \mathrm{C}$. Temperaturas alternadas favoreceram a germinação como a faixa de temperatura ótima determinada para cada espécie. As espécies são fotoblásticas positivas em ampla faixa de temperatura, indicando adaptação das mesmas a ambientes abertos, expostos ao sol.
\end{abstract}

Palavras-chave: Eriocaulaceae, Syngonanthus, germinação, luz, temperatura

ABSTRACT - (Light and temperature effects on seed germination of Syngonanthus elegantulus Ruhland, S. elegans (Bong.) Ruhland and S. venustus Silveira (Eriocaulaceae)). The objective of this study was evaluate light and temperature effects on seed germination of the seeds of Syngonanthus elegantulus Ruhland, S. elegans (Bong) Ruhland and S. venustus Silveira. The experiments were carried out in germination chambers with continuous light or dark and constant temperatures of $15,20,25,30,35$ and $40{ }^{\circ} \mathrm{C}$ and alternated temperatures of $25-15,30-15,35-15$ and $25-35^{\circ} \mathrm{C}$. The germination was low (36,5\%), under light, in S. elegantulus in the range 15 to $30^{\circ} \mathrm{C}$ and high in S. elegans $(75 \%)$ e S. venustus $(67,5 \%)$ from 20 to $30^{\circ} \mathrm{C}$. The temperature of $40{ }^{\circ} \mathrm{C}$ inhibited germination of all species. In S. elegantulus no germination occurred in the darkness, while in $S$. elegans low germination percentage $(11,5 \%)$ was observed at 25 and $30^{\circ} \mathrm{C}$ and in $S$. venustus (21\%), in the range 20 to $35^{\circ} \mathrm{C}$. Alternated temperatures favored seed germination as well as the range of constant temperatures defined from species. The species are positive photoblastic in a wide range of temperature, indicating adaptation for opened environments, with sun exposure.

Key words : Eriocaulaceae, Syngonanthus, germination, light, temperature

\section{Introdução}

Luz e temperatura são consideradas os dois principais fatores ambientais no controle da germinação (Baskin \& Baskin 1988; Bewley \& Black 1994; Benech-Arnold \& Sánchez 1995). Em geral, a luz é necessária para a germinação de sementes pequenas (Thompson \& Grime 1983; Bewley \& Black 1994, Rosa \& Ferreira 2001), cujas espécies estão associadas a ambientes abertos (Baskin \& Baskin 1971) ou perturbados (Fenner 1995). A relação entre germinação e temperatura está freqüentemente associada às temperaturas a que as plantas ficam expostas durante a fase de crescimento (Baskin \& Baskin 1988; 1992; Angosto \& Matilla 1993; Teketay 1998a; Villalobos \& Peláez 2001). A temperatura influi na velocidade e no percentual de germinação, especialmente por alterar a velocidade de absorção de água e modificar a velocidade das reações químicas que irão mobilizar ou degradar as reservas armazenadas e a síntese de várias substâncias para o crescimento das plântulas (Bewley \& Black 1994), ou provocar alterações na membrana plasmática (Thompson 1974; Hendricks \& Taylorson 1976). Dessa forma, estudos que abordam aspectos ecofisiológicos da germinação são essenciais para o entendimento do sucesso do estabelecimento das

\footnotetext{
Parte da Dissertação de Mestrado da primeira Autora

2 Universidade Federal de Minas Gerais, Instituto de Ciências Biológicas, Departamento de Botânica, Laboratório de Fisiologia Vegetal, C. Postal 486, CEP 30161-970, Belo Horizonte, MG, Brasil

3 Autor para correspondência: queila@dedalus.lcc.ufmg.br
} 
espécies em campo, que são determinados pela faixa de condições ambientais toleradas pelas sementes durante a germinação (Maluf \& Martins 1991).

As Eriocaulaceae são predominantemente herbáceas e apresentam distribuição pantropical, com maior diversidade de espécies nos campos rupestres de Minas Gerais e Bahia (Giulietti \& Hensold 1991). A família possui cerca de 1.200 espécies distribuídas em 13 gêneros, sendo o gênero Syngonanthus bem representado, com aproximadamente 180 espécies (Teixeira 1987). Em Minas Gerais, 17 espécies deste gênero estão relacionadas como ameaçadas de extinção (Mendonça \& Lins 2000), sendo S. elegans (Bong.) Ruhland (pé-de-ouro) uma das espécies mais exploradas comercialmente (Scatena et al. 1996), e S. venustus Silveira (brejeira), incluídas na categoria das espécies criticamente em perigo (Mendonça \& Lins 2000).

As espécies de eriocauláceaes são conhecidas popularmente como sempre-vivas (Teixeira 1987; Giulietti et al. 1988; 1996). A coleta indiscriminada, na qual as inflorescências são removidas antes da produção de sementes, compromete o recrutamento e diminui o tamanho das populações (Giulietti et al. 1988; 1996; Menezes \& Giulietti 2000). Entretanto, pouco se conhece sobre as características germinativas das sementes deste grupo de plantas. Entre os trabalhos disponíveis, estão os de Paepalanthus (Sá e Carvalho \& Ribeiro 1994 a; b; Kraus et al. 1996; Sano 1996) e de Syngonanthus (Scatena \& Menezes 1993; Scatena et al. 1996).

Devido a importância sócio-econômica e o potencial ornamental apresentado pelo gênero Syngonanthus, bem como sua vulnerabilidade frente à coleta predatória, este trabalho foi delineado objetivando avaliar a influência da luz e da temperatura sobre o comportamento germinativo de Syngonanthus elegantulus Ruhland, S. elegans (Bong.) Ruhland e $S$. venustus Silveira.

\section{Material e métodos}

Foram utilizadas sementes de Syngonanthus elegantulus Ruhland, coletadas no Parque Estadual do Rio Preto, município de São Gonçalo do Rio Preto (MG), e sementes de S. elegans (Bong.) Ruhland (péde-ouro) e $S$. venustus Silveira (brejeira), coletadas em Galheiros, distrito de Diamantina (MG), em julho/2001. Foram coletados escapos florais com capítulos bem desenvolvidos e maduros, estádio no qual as sementes possuem coloração avermelhada e apresentam-se livres entre si. No laboratório, os capítulos foram triturados em liqüidificador e peneirados, sendo as sementes separadas posteriormente com auxílio de microscópio estereoscópio.

Para a obtenção dos dados de massa foram utilizadas quatro repetições de 100 sementes por espécie, totalizando uma amostra de 400 sementes. Devido ao reduzido tamanho das sementes, a massa de matéria seca foi obtida pela pesagem de cada amostra (100 sementes) em balança analítica, após a secagem em estufa a $105^{\circ} \mathrm{C}$ até a estabilização do peso. O comprimento, considerado como a distância entre a base e o ápice, e a largura, foram medidos com auxílio de paquímetro digital (mm), em uma amostragem de 100 sementes por espécie.

Os testes de germinação foram realizados em câmaras de germinação sob luz $\left(30 \mu \mathrm{mol} \cdot \mathrm{m}^{-2} \cdot \mathrm{s}^{-1}\right)$ e escuro contínuos às temperaturas constantes de 15 , $20,25,30,35$ e $40{ }^{\circ} \mathrm{C}$. Foram utilizadas temperaturas alternadas de $25-15,30-15,35-15$ e $35-25^{\circ} \mathrm{C}(\mathrm{D} / \mathrm{N})$, em fotoperíodo de 12 horas, sendo que as temperaturas mais elevadas se referem ao período de luz. As sementes foram colocadas para germinar em placas de Petri forradas com folha dupla de papel de filtro umedecidas com solução de nistatina (100 UI/L) (Lemos Filho et al. 1997). A simulação da condição de escuro contínuo foi feita envolvendo-se as placas em papel alumínio e em sacos pretos de polietileno, sendo os experimentos montados e o acompanhamento feito em câmara escura, com luz verde de segurança (Souza \& Pereira 1992). Para todos os tratamentos foram utilizadas 200 sementes, distribuídas em quatro repetições de 50. A germinação foi avaliada diariamente, até a estabilização da resposta, com auxílio de um microscópio estereoscópio, sendo o critério para germinação a emergência do eixo embrionário (Scatena \& Menezes 1993).

Para análise estatística, os dados de germinação de $S$. elegantulus, expressos em percentagem, foram transformados em arco seno da raiz quadrada da percentagem e submetidos à análise de variância (ANOVA). As comparações entre as médias foram feitas mediante a aplicação do teste de Tukey ao nível de 5\% de probabilidade. Para S. elegans e S. venustus, como os dados não apresentaram homogeneidade, procedeu-se à análise não paramétrica de KruskalWallis, cujas ordenações médias foram então comparadas com o teste $\mathrm{T}(\alpha=0,05)$ (Sampaio 2002). A velocidade de germinação foi calculada segundo Labouriau (1970), para os tratamentos que atingiram valores acima de $10 \%$ de germinação. Para a 
comparação dos dados de germinabilidade e velocidade de germinação entre temperaturas constantes e alternadas foi realizada análise não paramétrica de Kruskal-Wallis cujas ordenações médias foram então comparadas com o teste $\mathrm{T}(\alpha=0,05)$. As medidas e a massa seca das sementes foram submetidos à análise de variância (ANOVA) e as comparações feitas pelo teste de Tukey ( $5 \%$ de probabilidade).

\section{Resultados}

As medidas de comprimento, largura e massa seca das sementes das espécies estudadas estão apresentadas na Tab. 1. Não houve diferença entre as medidas para sementes de S. elegantulus e S. elegans. Sementes de $S$. venustus apresentaram os maiores valores de comprimento e massa seca.

Syngonanthus elegantulus - As sementes desta espécie germinaram apenas na presença de luz. O percentual de germinação foi baixo (< 40\%) não diferindo na faixa de 15 a $30^{\circ} \mathrm{C}$. Não houve germinação a $40{ }^{\circ} \mathrm{C}$ (Tab. 2A). A velocidade de germinação aumentou gradativamente com a temperatura, sendo maior a $35^{\circ} \mathrm{C}$. A germinabilidade e a velocidade de germinação não diferiram entre as temperaturas alternadas de $25-15$ e $30-15^{\circ} \mathrm{C}$ (Tab. 2B). A germinação foi significativamente reduzida a $35-15$ e $35-25^{\circ} \mathrm{C}$, entretanto, a velocidade foi maior a $35-25^{\circ} \mathrm{C}$. As temperaturas alternadas de $25-15$ e $30-15^{\circ} \mathrm{C}$ foram tão efetivas em promover a germinação como a faixa de temperatura de 20 a $30^{\circ} \mathrm{C}(\mathrm{P}<0,05)$. A germinabilidade obtida a $35-15$ e $35-25^{\circ} \mathrm{C}$ foi similar à temperatura constante de $35^{\circ} \mathrm{C}$.

Syngonanthus elegans (pé de ouro) - A germinabilidade foi elevada ( $>50 \%$ ) e não diferiu na faixa de temperatura situada entre 20 a $30^{\circ} \mathrm{C}$, na presença da luz. A $40{ }^{\circ} \mathrm{C}$ o percentual de germinação foi muito

Tabela 1. Comprimento, largura e massa de matéria seca das sementes de Syngonanthus elegantulus Ruhland, S. elegans (Bong.) Ruhland e $S$. venustus Silveira (média \pm erro padrão; $n=100$ para comprimento e largura, e $n=4 \times 100$ para medida de massa seca).

\begin{tabular}{lccc}
\hline Espécies & $\begin{array}{c}\text { Comprimento } \\
(\mathrm{mm})\end{array}$ & $\begin{array}{c}\text { Largura } \\
(\mathrm{mm})\end{array}$ & $\begin{array}{c}\text { Massa seca } \\
(\mathrm{mg})\end{array}$ \\
\hline S. elegantulus & $0,36 \pm 0,04 \mathrm{a}$ & $0,21 \pm 0,02 \mathrm{a}$ & $1,3 \pm 0,00010 \mathrm{a}$ \\
S. elegans & $0,37 \pm 0,04 \mathrm{a}$ & $0,22 \pm 0,02 \mathrm{a}$ & $1,5 \pm 0,00025 \mathrm{a}$ \\
S. venustus & $0,50 \pm 0,04 \mathrm{~b}$ & $0,24 \pm 0,03 \mathrm{a}$ & $2,5 \pm 0,00013 \mathrm{~b}$ \\
\hline
\end{tabular}

Tabela 2. Valores médios de percentagem final (\%) e velocidade $\left(\mathrm{d}^{-1}\right)$ de germinação de sementes recém-coletadas de Syngonanthus elegantulus Ruhland sob luz e escuro contínuos em temperaturas constantes (A) e temperaturas alternadas (B), sendo que no par, as temperaturas mais elevadas se referem ao período de luz. Valores seguidos por letras iguais não diferem entre si, na mesma coluna, pelo teste de Tukey ao nível de 5\% de probabilidade.

A

\begin{tabular}{|c|c|c|c|c|}
\hline \multirow[t]{2}{*}{ Temperatura } & \multicolumn{2}{|c|}{$\begin{array}{c}\text { Germinação } \\
(\%)\end{array}$} & \multicolumn{2}{|c|}{$\begin{array}{c}\text { Velocidade média } \\
\qquad\left(\mathrm{d}^{-1}\right)\end{array}$} \\
\hline & $\begin{array}{c}\text { Luz } \\
\left(30 \mu \mathrm{mol} \cdot \mathrm{m}^{-2} \cdot \mathrm{s}^{-1}\right)\end{array}$ & Escuro & Luz & Escuro \\
\hline $15^{\circ} \mathrm{C}$ & $24,0 \mathrm{ab}$ & 0 & $0,046 \mathrm{c}$ & - \\
\hline $20^{\circ} \mathrm{C}$ & $36,5 \mathrm{a}$ & 0 & $0,056 \mathrm{c}$ & - \\
\hline $25^{\circ} \mathrm{C}$ & $35,5 \mathrm{a}$ & 0 & $0,078 \mathrm{~b}$ & - \\
\hline $30^{\circ} \mathrm{C}$ & $26,5 \mathrm{ab}$ & 0 & $0,084 \mathrm{~b}$ & - \\
\hline $35^{\circ} \mathrm{C}$ & $15,0 \mathrm{~b}$ & 0 & $0,111 \mathrm{a}$ & - \\
\hline $40^{\circ} \mathrm{C}$ & $0,0 \mathrm{c}$ & 0 & - & - \\
\hline
\end{tabular}

B

\begin{tabular}{llc} 
Temperatura & $\begin{array}{c}\text { Germinação } \\
(\%)\end{array}$ & $\begin{array}{c}\text { Velocidade média } \\
\left(\mathrm{d}^{-1}\right)\end{array}$ \\
\hline $25-15^{\circ} \mathrm{C}$ & $44,5 \mathrm{a}$ & $0,065 \mathrm{bc}$ \\
$30-15^{\circ} \mathrm{C}$ & $40,5 \mathrm{ab}$ & $0,067 \mathrm{~b}$ \\
$35-15{ }^{\circ} \mathrm{C}$ & $20,0 \mathrm{bc}$ & $0,049 \mathrm{c}$ \\
$35-25{ }^{\circ} \mathrm{C}$ & $17,0 \mathrm{c}$ & $0,093 \mathrm{a}$ \\
\hline
\end{tabular}

baixo (1\%) (Tab. 3A). A velocidade de germinação foi semelhante na faixa de 25 a $35^{\circ} \mathrm{C}$. No escuro, a germinação foi muito baixa $(<12 \%)$. Não houve diferença na percentagem e velocidade de germinação entre os pares $25-15,30-15$ e $35-15^{\circ} \mathrm{C}$, sendo a germinação significativamente reduzida a $35-25^{\circ} \mathrm{C}$ (Tab. 3B). A germinabilidade obtida a $30-15$ e $35-15^{\circ} \mathrm{C}$ foi semelhante às temperaturas constantes de 20 a $30^{\circ} \mathrm{C}(\mathrm{P}<0,05)$. O percentual de germinação no regime alternado de $35-25{ }^{\circ} \mathrm{C}$ não diferiu dos valores encontrados em 15 e $35^{\circ} \mathrm{C}$.

Syngonanthus venustus (brejeira) - A percentagem final de germinação, sob luz, foi elevada (> 50\%) e semelhante na faixa de 20 a $30{ }^{\circ} \mathrm{C}$, não havendo germinação a $40^{\circ} \mathrm{C}$ (Tab. $\left.4 \mathrm{~A}\right)$. A germinação foi mais rápida na faixa de 25 a $35^{\circ} \mathrm{C}$. No escuro, a germinabilidade foi sempre menor que na presença de luz. Temperaturas extremas de 15 e $40^{\circ} \mathrm{C}$ inibiram a germinação. Os valores de percentagem e velocidade de germinação não apresentaram diferenças significativas nas combinações $25-15,30-15$ e $35-15^{\circ} \mathrm{C}$, no entanto, a percentagem de germinação diminuiu, nos pares $35-15$ e $35-25^{\circ} \mathrm{C}$ e neste último par a 
Tabela 3. Valores médios de percentagem final (\%) e velocidade $\left(\mathrm{d}^{-1}\right)$ de germinação de sementes recém-coletadas de Syngonanthus elegans (Bong.) Ruhland sob luz e escuro contínuos em temperaturas constantes (A) e temperaturas alternadas (B), sendo que no par, as temperaturas mais elevadas se referem ao período de luz. Valores seguidos por letras iguais não diferem entre si, na mesma coluna, pelo teste $\mathrm{T}$ ao nível de $5 \%$ de probabilidade.

A

\begin{tabular}{|c|c|c|c|c|}
\hline \multirow[t]{2}{*}{ Temperatura } & \multicolumn{2}{|c|}{$\begin{array}{c}\text { Germinação } \\
(\%)\end{array}$} & \multicolumn{2}{|c|}{$\begin{array}{c}\text { Velocidade média } \\
\qquad\left(\mathrm{d}^{-1}\right)\end{array}$} \\
\hline & $\begin{array}{c}\text { Luz } \\
\left(30 \mu \mathrm{mol} \cdot \mathrm{m}^{-2} \cdot \mathrm{s}^{-1}\right)\end{array}$ & Escuro & Luz & Escuro \\
\hline $15^{\circ} \mathrm{C}$ & $55,0 \mathrm{~b}$ & $1,0 \mathrm{c}$ & $0,059 \mathrm{~b}$ & - \\
\hline $20^{\circ} \mathrm{C}$ & $68,5 \mathrm{ab}$ & $1,0 \mathrm{c}$ & $0,083 \mathrm{~b}$ & - \\
\hline $25^{\circ} \mathrm{C}$ & $72,0 \mathrm{ab}$ & $4,0 \mathrm{~b}$ & $0,183 \mathrm{a}$ & - \\
\hline $30^{\circ} \mathrm{C}$ & $75,0 \mathrm{a}$ & $11,5 \mathrm{a}$ & $0,226 \mathrm{a}$ & 0,102 \\
\hline $35^{\circ} \mathrm{C}$ & $59,0 \mathrm{~b}$ & $3,0 \mathrm{c}$ & 0,252 a & - \\
\hline $40^{\circ} \mathrm{C}$ & $1,0 \mathrm{c}$ & $0,0 \mathrm{c}$ & - & - \\
\hline
\end{tabular}

B

\begin{tabular}{lcc} 
Temperatura & $\begin{array}{c}\text { Germinação } \\
(\%)\end{array}$ & $\begin{array}{c}\text { Velocidade média } \\
\left(\mathrm{d}^{-1}\right)\end{array}$ \\
\hline $25-15{ }^{\circ} \mathrm{C}$ & $83,5 \mathrm{a}$ & $0,170 \mathrm{~b}$ \\
$30-15^{\circ} \mathrm{C}$ & $82,5 \mathrm{a}$ & $0,144 \mathrm{~b}$ \\
$35-15^{\circ} \mathrm{C}$ & $80,0 \mathrm{a}$ & $0,177 \mathrm{~b}$ \\
$35-25^{\circ} \mathrm{C}$ & $55,0 \mathrm{~b}$ & $0,207 \mathrm{a}$ \\
\hline
\end{tabular}

germinação foi mais rápida (Tab. 4B). Os regimes alternados de temperatura de $25-15$ e $30-15^{\circ} \mathrm{C}$ favoreceram a germinação assim como a faixa de 20 a $30{ }^{\circ} \mathrm{C}$. A percentagem de germinação sob $35-25^{\circ} \mathrm{C}$ não diferiu da obtida sob as temperaturas constantes de 15 e $35^{\circ} \mathrm{C}$.

\section{Discussão}

Sementes pequenas, em geral, necessitam de luz para germinar (Thompson \& Grime 1983; Bewley \& Black 1994; Rosa \& Ferreira 2001) e o tamanho das sementes pode estar relacionado às condições ambientais mais favoráveis ao estabelecimento da plântula (Westoby et al. 1992). Sementes grandes estão associadas com hábitats sombreados (Leishman et al. 2000), enquanto as de pequeno tamanho são associadas com hábitats abertos (Leishman \& Westoby 1994; Seiwa \& Kikuzawa 1996). Neste estudo, as sementes das espécies investigadas podem ser consideradas muito pequenas, possuindo comprimento médio menor que $1 \mathrm{~mm}$ (sensu Ekstam et al. 1999) e massa seca menor que 0,1 mg (sensu Hughes et al. 1994); apresentaram
Tabela 4. Valores médios de percentagem final (\%) e velocidade $\left(\mathrm{d}^{-1}\right)$ de germinação de sementes recém-coletadas de Syngonanthus venustus Silveira sob luz e escuro contínuos em temperaturas constantes (A) e temperaturas alternadas (B), sendo que no par, as temperaturas mais elevadas se referem ao período de luz. Valores seguidos por letras iguais não diferem entre si, na mesma coluna, pelo teste $\mathrm{T}$ ao nível de $5 \%$ de probabilidade.

A

\begin{tabular}{|c|c|c|c|c|}
\hline \multirow[t]{2}{*}{ Temperatura } & \multicolumn{2}{|c|}{$\begin{array}{c}\text { Germinação } \\
(\%)\end{array}$} & \multicolumn{2}{|c|}{$\begin{array}{c}\text { Velocidade média } \\
\qquad\left(\mathrm{d}^{-1}\right)\end{array}$} \\
\hline & $\begin{array}{c}\text { Luz } \\
\left(30 \mu \mathrm{mol} \cdot \mathrm{m}^{-2} \cdot \mathrm{s}^{-1}\right)\end{array}$ & Escuro & Luz & Escuro \\
\hline $15^{\circ} \mathrm{C}$ & $49,0 \mathrm{~b}$ & $0,0 \mathrm{c}$ & $0,063 \mathrm{~b}$ & - \\
\hline $20^{\circ} \mathrm{C}$ & $67,5 \mathrm{a}$ & $9,5 \mathrm{~b}$ & $0,098 \mathrm{~b}$ & - \\
\hline $25^{\circ} \mathrm{C}$ & $65,5 \mathrm{a}$ & $6,5 \mathrm{~b}$ & $0,181 \mathrm{a}$ & - \\
\hline $30^{\circ} \mathrm{C}$ & $67,0 \mathrm{a}$ & $21,0 \mathrm{a}$ & $0,268 \mathrm{a}$ & 0,124 \\
\hline $35^{\circ} \mathrm{C}$ & $43,5 \mathrm{~b}$ & $12,0 \mathrm{~b}$ & $0,230 \mathrm{a}$ & 0,129 \\
\hline $40^{\circ} \mathrm{C}$ & $0,0 \mathrm{c}$ & $1,0 \mathrm{c}$ & - & - \\
\hline
\end{tabular}

B

\begin{tabular}{llc} 
Temperatura & $\begin{array}{c}\text { Germinação } \\
(\%)\end{array}$ & $\begin{array}{c}\text { Velocidade média } \\
\left(\mathrm{d}^{-1}\right)\end{array}$ \\
\hline $25-15^{\circ} \mathrm{C}$ & $69,0 \mathrm{a}$ & $0,142 \mathrm{~b}$ \\
$30-15{ }^{\circ} \mathrm{C}$ & $65,5 \mathrm{a}$ & $0,157 \mathrm{~b}$ \\
$35-15^{\circ} \mathrm{C}$ & $55,5 \mathrm{ab}$ & $0,149 \mathrm{~b}$ \\
$35-25^{\circ} \mathrm{C}$ & $45,0 \mathrm{~b}$ & $0,230 \mathrm{a}$ \\
\hline
\end{tabular}

maior percentagem de germinação na presença da luz sendo, portanto, fotoblásticas positivas, em ampla faixa de temperatura. $\mathrm{O}$ comportamento fotoblástico positivo observado para as espécies de Syngonanthus no presente estudo é similar ao descrito para algumas espécies de ocorrência nos campos rupestres, como em Eriocaulaceae (Sá e Carvalho \& Ribeiro 1994a; b; Scatena et al. 1996), Velloziaceae (Mercier \& Guerreiro Filho 1989; Garcia \& Diniz 2003) e Xyridaceae (Abreu \& Garcia 2005).

Outra característica relacionada à exigência de luz é o fato de que após a emergência do eixo embrionário, algumas espécies de Eriocaulaceae desenvolvem primeiramente as folhas e posteriormente as raízes adventícias (Scatena et al. 1993; 1996; Kraus et al. 1996). As espécies de Syngonanthus crescem em diferentes condições edáficas, habitando solos secos, úmidos e até alagados, mas sempre expostas ao sol (Scatena et al. 1999). Assim, para o gênero Syngonanthus, o pequeno tamanho das sementes e a necessidade de luz para germinação podem ser considerados estratégias adaptativas a ambientes abertos, expostos ao sol, como os campos rupestres. 
A percentagem de germinação de $S$. elegantulus foi baixa (máxima de $36,5 \%$ ) na faixa de 15 a $30^{\circ} \mathrm{C}$. Em S. elegans e $S$. venustus entre 20 a $30{ }^{\circ} \mathrm{C}$ foi registrado o percentual mais alto de germinação ( $75 \%$ e $67,5 \%$ respectivamente). Estudos realizados com espécies de Velloziaceae (Mercier \& Guereiro Filho 1989; Garcia \& Diniz 2003), típicas de campos rupestres, registraram altas porcentagens de germinação em ampla faixa de temperatura, sendo que Vellozia gigantea N.L. Menezes \& Mello-Silva, $V$. glandulifera Goethart \& Henrard e V. variabilis Mart. ex Schult. \& S. Schult. (Garcia \& Diniz 2003) apresentaram porcentagens elevadas também sob temperaturas mais altas $\left(35\right.$ e $\left.40^{\circ} \mathrm{C}\right)$. A temperatura de $25^{\circ} \mathrm{C}$ pode ser considerada ótima para germinação de $S$. elegantulus e a de $30^{\circ} \mathrm{C}$ para S. elegans e $S$. venustus, visto que nestas temperaturas as sementes apresentaram germinação mais rápida e em maior percentagem. A correlação positiva existente entre percentagem e velocidade de germinação das sementes garante sucesso no desenvolvimento e estabelecimento de novas plantas e pode também auxiliar no plano de manejo para o cultivo das espécies (Scatena et al. 1996).

Em geral, sementes pequenas respondem ao regime de alternância de temperatura, uma vez que são encontradas próximas à superfície do solo, região onde as flutuações térmicas são frequientes (Thompson \& Grime 1983; Thompson 1993). Entretanto, para as espécies de Syngonanthus estudadas as flutuações de temperatura foram tão efetivas em promover a germinação das sementes como a faixa ótima de temperaturas constantes observada para cada espécie, sugerindo que o fator preponderante em promover a germinação das sementes destas espécies é a presença de luz. A exigência de temperaturas alternadas para a germinação de sementes está associada a tipos particulares de habitat (Raybould et al. 1977). Teketay (1998b) verificou que a germinação de duas espécies de Veronica (Schrophulariaceae), que produzem sementes muito pequenas $(0,1 \mathrm{mg})$ e são típicas de ambientes perturbados foram dependentes de luz e regimes alternados de temperatura. Várias espécies européias, típicas de habitats alagados, tiveram a germinação de suas sementes promovidas pela luz e flutuações de temperatura, embora algumas necessitam do escuro com alternância para germinação (Thompson \& Grime 1983). De modo geral as alternâncias de temperatura apenas favorecem a germinação quando as sementes não germinam sob temperaturas constantes, indicando a adaptação das espécies ao ambiente em que se encontram (Thompson 1974; 1993; Thompson \& Grime 1983).

No presente estudo, a germinação de S. elegantulus foi reduzida nos pares de temperatura onde $35^{\circ} \mathrm{C}$ estava presente. A alternância favoreceu a germinação $S$. elegans quando nos pares de temperatura foi incluída a de $15^{\circ} \mathrm{C}$, enquanto a alternância de duas temperaturas constantes mais elevadas $\left(25-35{ }^{\circ} \mathrm{C}\right)$, reduziu a germinação. A germinação de $S$. venustus aumentou sob temperaturas alternadas na qual foi incluída a temperatura de $15{ }^{\circ} \mathrm{C}$, mostrando comportamento similar a S. elegans, porém, com menor velocidade, e diminuiu nos pares onde foi introduzida a temperatura de $35^{\circ} \mathrm{C}$. Comportamento semelhante foi observado para as sementes de S. elegantulus.

S. elegans apresenta grandes populações e ampla área de distribuição, desde Diamantina até a Serra do Cipó (Giulietti et al. 1988; 1996; Parra 1998). Ocorre em hábitats xéricos (Scatena et al. 1999), o que poderia favorecer a germinação das sementes sob temperaturas mais elevadas. S. elegantulus que ocorre ao longo da Cadeia do Espinhaço em Minas Gerais, desde o município de Grão Mogol até a Serra do Cipó, onde apresenta pequenas populações em poucas localidades (Parra 1998). Foi encontrada também em áreas planas, com solos arenosos entremeados por bolsões mais úmidos (observação pessoal). S. venustus ocorre no planalto de Diamantina e na Serra do Cabral (Giulietti et al. 1988; 1996), é uma espécie típica de hábitats brejosos, do qual originouse o codinome brejeira.

$\mathrm{O}$ fato das altas temperaturas inibirem o processo germinativo de $S$. elegantulus e $S$. venustus, pode estar relacionado com a ocorrência das mesmas em hábitats alagados. Alta umidade dos solos causa diminuição na amplitude de flutuações térmicas (Klips \& Peñalosa 2003), o que pode afetar a resposta germinativa das sementes destas espécies. Assim, alternâncias de temperaturas com valores mais elevados durante o dia e mais baixos durante a noite podem favorecer a germinação de sementes de $S$. elegans (ambientes xéricos), enquanto que temperaturas mais elevadas, durante o dia, reduzem a germinação de $S$. elegantulus e de $S$. venustus (ambientes úmidos).

\section{Agradecimentos}

À CAPES, pela concessão da bolsa à primeira Autora; ao Prof. Dr. Ivan Barbosa Machado Sampaio, pelo auxílio na revisão da análise estatística dos dados; 
ao Msc. Fábio Vieira, Dr. José Pires Lemos Filho, Dr. Amaury Alves Alvarenga e aos assessores da Acta Botanica Brasilica, pela leitura crítica do manuscrito e sugestões.

\section{Referências bibliográficas}

Abreu, M.E.P. \& Garcia, Q.S. 2005. Efeito da luz e da temperatura na germinação de sementes de quatro espécies de Xyris L. (Xyridaceae) ocorrentes na Serra do Cipó, MG, Brasil. Acta Botanica Brasilica 19(1): 149-154.

Angosto, T. \& Matilla, A.J. 1993. Variations in seeds of three endemic leguminous species at different altitudes. Physiologia Plantarum 87: 329-334.

Baskin, J.M. \& Baskin, C.C. 1971. The possible ecological significance of the light requirement for germination in Cyperus inflexus. Bulletin of the Torrey Botanical Club 98: $25-33$.

Baskin, J.M. \& Baskin, C.C. 1988. Germination ecophysiology herbaceous plant species in a temperature region. American Journal of Botany 75: 286-305.

Baskin, J.M. \& Baskin, C.C. 1992. Role of temperature and light in the germination ecology of buried seeds of weedy species of disturbed forests. I. Lobelia inflata. Canadian Journal of Botany 70: 589-592.

Bewley, J.D. \& Black, M. 1994. Seeds: Physiology of Development and Germination. $2^{\text {nd }}$ ed., New York, Plenum Press.

Benech-Arnold, R. \& Sánchez, R.A. 1995. Modeling weed seed germination. Pp. 545-566. In: J. Kigel \& G. Galili (eds.). Seed development and germination. New York, Academic Press.

Ekstan, B.; Johannessson, R.M. \& Milberg, P. 1999. The effect of light and number of diurnal temperature fluctuations on germination of Phragmites australis. Seed Science Research 9: 165-170.

Fenner, M. 1995. Ecology of seed banks Pp. 507-543. In: J. Kigel \& G. Galili (eds.). Seed development and germination. New York, Academic Press.

Garcia, Q.S. \& Diniz, I.S.S. 2003. Comportamento germinativo de três espécies de Vellozia da Serra do Cipó (MG). Acta Botanica Brasilica 17: 487-494.

Giulietti, A.M. \& Hensold, N.C. 1991. Synonymization of the genera Comanthera and Carptotepala with Syngonanthus (Eriocaulaceae). Annals of the Missouri Botanical Garden 78: 273-295.

Giulietti, A.M.; Wanderley, M.G.L.; Longhi-Wagner, H.M.; Pirani, J.R. \& Parra, J.R. 1996. Estudos em "semprevivas": taxonomia com ênfase nas espécies de Minas Gerais, Brasil. Acta Botanica Brasilica 10: 329-384.

Giulietti, N.; Giulietti, A.M.; Pirani, J.R. \& Menezes, N.L. 1988. Estudos de sempre-vivas: importância econômica do extrativismo em Minas Gerais, Brasil. Acta Botanica Brasilica 1: 179-194.

Hendricks, S.B. \& Taylorson, R.B. 1976. Variation in germination and amino acid leakage of seed with temperature related to membrane phase change. Plant Physiology 58: 7-11.
Hughes, L.; Dunlop, M.; French, K.; Leishman, M.R.; Rice, B.; Rodgerson, L. \& Westoby, M. 1994. Predicting dispersal spectra: a minimal set of hypotheses based on plant attribute. Journal of Ecology 82: 933-950.

Klips, A.R. \& Peñalosa, J. 2003. The timing of seed fall, innate dormancy, and ambient temperature in Lythrum salicaria. Aquatic Botany 75: 1-7.

Kraus, J.E.; Scatena, V.L.; Lewinger, M.E. \& Sá Trench, K.U. 1996. Morfologia externa e interna de quatro espécies de Paepalanthus Kunth (Eriocaulaceae) em desenvolvimento pós-seminal. Boletim de Botânica da Universidade se São Paulo 15: 45-53.

Labouriau, L.F.G. 1970. On the physiology of seed germination in Vicia graminea Sm I. Anais da Academia Brasileira de Ciências 42: 235-262.

Leishman, M.R. \& Westoby, M. 1994. The role of large seed size in shaded conditions: experimental evidence. Functional Ecology 8: 205-214.

Leishman, M.R.; Wright, I.J.; Moles, A.T. \& Westoby, M. 2000. The evolutionary ecology of seed size. Pp. 31-57. In: M. Fenner (ed.). The ecology of regeneration in plant communities. $2^{\text {nd }}$ ed. CABI International Wallingford UK.

Lemos Filho, J.P.; Guerra, S.T.M.; Lovato, M.B. \& Scotti, M.R.M.M.L. 1997. Germinação de sementes de Senna macranthera, Senna multifuga e Stryphnodendron polyphyllum. Pesquisa Agropecuária Brasileira 32: 357-361.

Maluf, A.M. \& Martins, P.S. 1991. Germinação de sementes de Amaranthus hybridus L. e Amaranthus viridis L. Revista Brasileira de Biologia 51: 417-425.

Mendonça, M.P. \& Lins, L.V. 2000. Lista vermelha das espécies ameaçadas de extinção da flora de Minas Gerais. Minas Gerais, Belo Horizonte, Fundação Biodiversitas \& Fundação Zoo-Botânica de Belo Horizonte.

Menezes, N.Z. \& Giulietti, A.M. 2000. Campos rupestres. Pp. 65-73. In: M.P. Mendonça \& L.V. Lins (eds.). Lista vermelha das espécies ameaçadas de extinção da flora de Minas Gerais. Minas Gerais, Belo Horizonte, Fundação Biodiversitas \& Fundação Zoo-Botânica de Belo Horizonte.

Mercier, H. \& Guerreiro-Filho, O. 1989. Germinação de Pleurostima fanniei Menezes, P. rogieri (Hort, ex Moore \& Ayres) Menezes e Vellozia alata L.B. Smith (Velloziaceae) sob diferentes condições de temperatura. Hoehnea 16: 195-202.

Parra, J.R. 1998. Flora da serra do Cipó, Minas Gerais: Syngonanthus Ruhland (Eriocaulaceae). Boletim de Botânica da Universidade de São Paulo 17: 219-254.

Raybould, C.C.; Unsworth, M.H. \& Gregory, P.J. 1977. Seed germination in response to diurnal fluctuations of temperature. Nature 267: 147-149.

Rosa, S.G.T. \& Ferreira, A.G. 2001. Germinação de sementes de plantas medicinais lenhosas. Acta Botanica Brasilica 15: $147-288$.

Sá e Carvalho, C.G. \& Ribeiro, M.C. 1994a. Efeitos do armazenamento e de reguladores de crescimento na germinação de Paepalanthus speciosus, Eriocaulaceae. Revista Brasileira de Botânica 17: 61-65. 
Sá e Carvalho, C.G. \& Ribeiro, M.C. 1994b. Efeitos de choques térmicos na germinação de Paepalanthus speciosus Koern. (Eriocaulaceae). Acta Botanica Brasilica 8: 205-211.

Sampaio, I.B.M. 2002. Estatística aplicada a experimentação animal. Belo Horizonte, Fundação de Ensino e Pesquisa em Medicina.

Sano, P.T. 1996. Fenologia de Paepalanthus hilairei Koren., $P$. polyanthus (Bong.) Kunth e $P$. robustus Silveira: Paepalanthus sect. Actinocephalus Koern. Eriocaulaceae. Acta Botanica Brasilica 10: 317-327.

Scatena, V.L. \& Menezes, N.L. 1993. Embryology and seedling development in Syngonanthus rufipes Silveira (Eriocaulaceae). Beitraege Zur Biologie der Pflanzen 67: 333-343

Scatena, V.L.; Cardoso, V.A. \& Giulietti, A.M. 1999. Morfoanatomia de espécies de Blastocaulon Ruhland (Eriocaulaceae). Acta Botanica Brasilica 13: 29-41.

Scatena, V.L.; Lemos, F.J.P. \& Lima, A.A.A. 1996. Morfologia do desenvolvimento pós-seminal de Syngonanthus elegans e $S$. niveus (Eriocaulaceae). Acta Botanica Brasilica 10: 85-91.

Seiwa, K. \& Kikuzawa, K. 1996. Importance of seed size for the establishment of seedlings of five deciduous broadleaved tree species. Vegetation 123: 51-64.

Souza R.P. \& Pereira, M.F.D.A. 1992. Interação de luz, GA 3 e estratificação na germinação de sementes de Impatiens wallerana. Revista Brasileira de Fisiologia Vegetal 4 : 21-25.
Teixeira, A.M. 1987. Sempre-vivas: folclore e verdade. Ciência Hoje 5: 14-15.

Teketay, D. 1998a. Germination of Acacia origena, A. pilispina and Pterolobium stellatum in response to different pre-sowing seed treatments, temperature and light. Journal of Arid Environments 38: 551-560.

Teketay, D. 1998b. The joint role of alternanting temperatures and light quality in the germination of Veronica anagallis-aquatica and V. javanica. Tropical Ecology 39: 179-184.

Thompson, K. 1993. Germination at alternating temperatures. Pp. 176-205. In: G.A.F. Hendry \& J.P. Grime (eds.). Methods in comparative plant ecology. London, Champman \& Hall.

Thompson, K. \& Grime, J.P. 1983. A comparative study of germination responses to diurnally-fluctuating temperatures. Journal of Applied Ecology 20: 141-156.

Thompson, P.A. 1974. Effects of fluctuating temperature on germination. Journal of Experimental Botany 25: 164-175.

Villalobos, A.E. \& Peláez, D.V. 2001. Influences of temperature and water stress on germination and establishment of Prosopis caldenia Burk. Journal of Arid Environments 49: 321-328.

Westoby, M.; Jurado, E. \& Leishman, M. 1992. Comparative evolutionary ecology of seed size. Tree 7: 368-372. 